\title{
Development and validation of a nomogram to predict the mortality risk in elderly patients with ARF
}

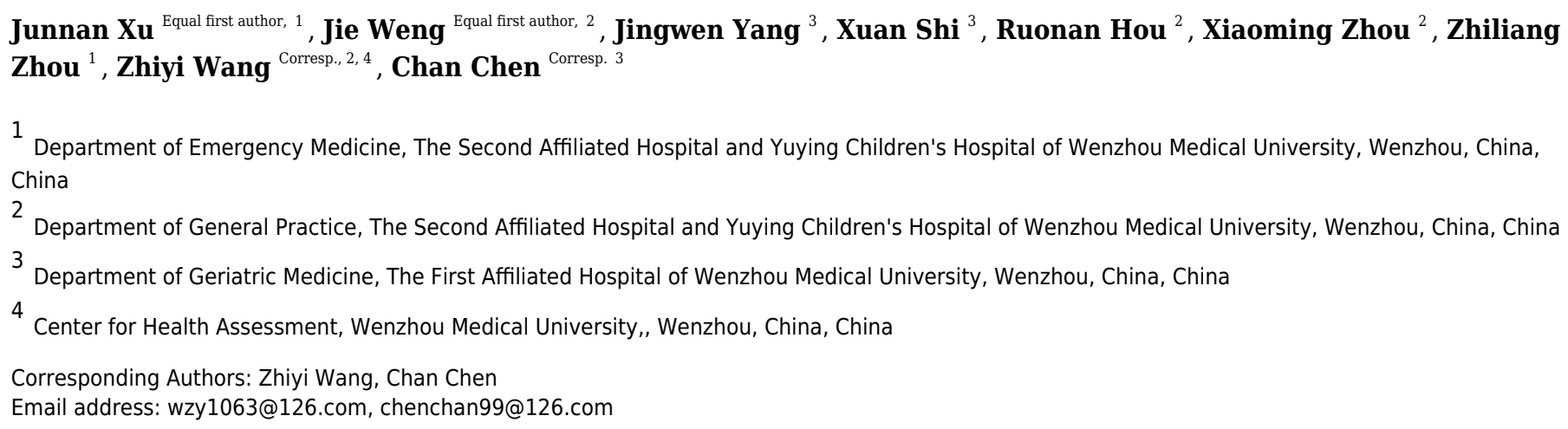

Background: Acute respiratory failure (ARF) is a life-threatening complication in elderly patients. We developed a nomogram model to explore the risk factors of prognosis and the short-term mortality in elderly patients with ARF. Methods: 759 patients from MIMIC-III database were categorized into the training set and 673 patients from our hospital were categorized into the validation set. Demographical, laboratory variables, SOFA score and APS-III score were collected within the first $24 \mathrm{~h}$ after the ICU admission. A 30-day followup was performed for all patients. Results: Multivariate logistic regression analysis showed that the heart rate, respiratoryrate, systolic pressure, $\mathrm{SPO}_{2}$, albumin and $24 \mathrm{~h}$ urine output were independent prognostic factors for 30-day mortality in ARF patients. A nomogram was established based on above independent prognostic factors. This nomogram had C-index of 0.741 (95\% Cl: $0.7058-0.7766)$, and the C-index was 0.687 (95\% Cl: $0.6458-0.7272)$ in the validation set. The calibration curves both in training and validation set were close to the ideal model. The SOFA had a C-index of 0.653 and the APSIII had a C-index of 0.707 in predicting 30-day mortality. Conclusion: Our nomogram performed better than APS-III and SOFA scores and should be useful as decision support on the prediction of mortality risk in elderly patients with ARF. 


\section{Development and validation of a nomogram to predict the mortality risk}

\section{2 in elderly patients with acute respiratory failure}

3 Xu Junnan ${ }^{1 *}$, Weng Jie ${ }^{2 *}$, Yang Jingwen ${ }^{3}$, Shi Xuan ${ }^{3}$, Hou Ruonan ${ }^{2}$, Zhou Xiaoming ${ }^{2}$, Zhou

4 Zhiliang ${ }^{1}$, Wang Zhiyi ${ }^{2,4 \#}$ Chen Chan ${ }^{3 \#}$

$5{ }^{1}$ Department of Emergency Medicine, The Second Affiliated Hospital and Yuying Children's

6 Hospital of Wenzhou Medical University, Wenzhou, 325027, China; ${ }^{2}$ Department of General

7 Practice, The Second Affiliated Hospital and Yuying Children's Hospital of Wenzhou Medical

8 University, Wenzhou, 325027, China; ${ }^{3}$ Department of Geriatric Medicine, The First Affiliated

9 Hospital, Wenzhou Medical University, Wenzhou 325000, China; ${ }^{4}$ Center for Health Assessment,

10 Wenzhou Medical University, Wenzhou, 325000, China.

11 *X.J. and W.J. contributed equally to this work

12 \#Correspondence to: Wang Zhiyi,wzy1063@126.cm; Chen Chan, chenchan99@126.com

\section{ABSTRACT}

Background: Acute respiratory failure (ARF) is a life-threatening complication in elderly patients. We developed a nomogram model to explore the risk factors of prognosis and the shortterm mortality in elderly patients with ARF.

Methods: A total of 759 patients from MIMIC-III database were categorized into the training set and 673 patients from our hospital were categorized into the validation set. Demographical, laboratory variables, SOFA score and APS-III score were collected within the first $24 \mathrm{~h}$ after the ICU admission. A 30-day follow-up was performed for all patients.

Results: Multivariable logistic regression analysis showed that the heart rate, respiratory rate, systolic pressure, $\mathrm{SPO}_{2}$, albumin and $24 \mathrm{~h}$ urine output were independent prognostic factors for 30-day mortality in ARF patients. A nomogram, elderly ARF mortality prediction nomogram (eARF-MPN), was established based on above independent prognostic factors. This e-ARF-MPN had concordance index (C-index) of 0.741 (95\% CI: 0.706-0.777), and the C-index was 0.687 
(95\%CI: 0.646-0.727) in the validation set. The calibration curves both in training and validation set were close to the ideal model. The SOFA had a C-index of 0.653 and the APS-III had a Cindex of 0.707 in predicting 30 -day mortality.

Conclusion: Our e-ARF-MPN performed better than APS-III and SOFA scores and could be useful as decision support on the prediction of mortality risk in elderly patients with ARF.

Keywords: Acute respiratory failure; Mortality risk; Prognosis; Nomogram

\section{INTRODUCTION}

Acute respiratory failure $(\mathrm{ARF})$ is a common complication of critically ill patients. With an ageing population there is a growing prevalence of ARF. The incidence of ARF in the 65-84 age group was approximately twice that of the 55-64 age group and more than three times that of the young age group(Flaatten et al. 2017). In addition, ARF in elderly patients is associated with a high mortality rate(Behrendt 2000). The reasons included the heterogeneity and complexity of the elderly patient condition. Therefore, accurate assessment of the severity of ARF in the elderly patients is the key to reduce its mortality.

Academic research shows that clinical signs of ARF, including hypercapnia $>45 \mathrm{mmHg}$, clearance of creatinine $<50 \mathrm{ml}$ minute-1, elevated NT-pro-B-type natriuretic peptide or B-type natriuretic peptide, were predictive of death(Ray et al. 2006). Although the risk factors are clear, there is still no consensus about the prognostic factors and evaluation system.

Some studies report that Simplified Acute Physiology Score-III(Gannon et al. 2018) and SOFA score(Dziadzko et al. 2018) can identify patients at high risk of death as reliably as the early warning score. with ARF. However, the sensitivity and specificity of these tools prediction are unsatisfactory. Moreover, it includes multiple indicators which are cumbersome to calculate. At present, there is a lack of clinical prediction tools of death for ARF in the elderly, therefore, it is urgent to find a risk stratification tool to predict mortality in elderly patients with ARF. The nomogram model can quantify, graph, and visualize Logistic regression results to achieve individualized prediction of disease risk. It has been successfully used in clinical diagnosis and prognostic assessment of various diseases(Dong et al. 2018; Gilbride et al. 2018). 
In this study, we analyze the prognostic factors of elderly ARF patients, construct a nomogram model to predict survival, evaluate the risk of death of elderly patients, and then provide clinical help for early identification and intervention of high-risk patients to improve their prognosis.

\section{MATERIALS \& METHODS}

\section{Database and subjects}

Subject data were retrieved from Medical Information Mart for Intensive Care III database version 1.4 (MIMIC-III v1.4) and the Second Affiliated Hospital and Yuying Children's Hospital of Wenzhou Medical University in China. The MIMIC-III is a clinical database comprising the information of 46,520 patients who were admitted to the ICU of Beth Israel Deaconess Medical Center (BIDMC) in Boston, MA, from 2001 to 2012(Johnson et al. 2016). MIMIC-III is a large, freely accessible database for international researchers upon a use agreement (Our certification number: 31355221). The database was approved by the institutional review boards (IRB) of the Massachusetts Institute of Technology (MIT) and BIDMC, and consent was obtained for the original data collection (unidentified health information of patients was used); therefore, the informed consent was waived in our study. The MIT and BIDMC are responsible for waiving the informed consent.

\section{Participants}

ARF was identified from ICD-9 code in the MIMIC-III database. For patients with multiple ICU admissions, we included only the first ICU admission. The primary outcome in this study was patients' 30 -day mortality (died within 30 days after hospitalization).

\section{Data extraction}

Demographical and laboratory variables were extracted from MIMIC-III database by Structure Query Language (SQL) at the first $24 \mathrm{~h}$ of ICU admission. We collected the following data: age, gender, vital signs, mechanical ventilation (including invasive and non-invasive), percutaneous oxygen saturation (SpO2), white blood cell (WBC), hemoglobin, platelet, albumin, bilirubin, blood urea nitrogen (BUN), lactate, activated partial thromboplastin time (APTT), prothrombine time (PT), 24 hours urine output, sequential organ failure assessment (SOFA) score, and Acute 
Physiology Score III (APS-III). If the laboratory variables were examined more than once, the greatest severity value associated with the illness was used in our study. SOFA and APS-III scores were calculated within the first $24 \mathrm{~h}$ after the ICU admission. Patients with missing data, length of stay in ICU $<24 \mathrm{~h}$, and who were younger than 60 years old(Lloyd-Sherlock et al. 2012) were excluded from this study. Finally, 759 ARF patients were included in our analysis. All the scripts used to calculate the SOFA and APS-III scores were available from GitHub website (https://github.com/MIT-LCP/mimic-code/tree/master/concepts). Meanwhile, the same clinical data of ARF patients admitted to ICU in the Second Affiliated Hospital of Wenzhou Medical University from January 2010 to January 2020 were collected retrospectively. Inclusion criteria were length of stay in ICU $\geq 24 \mathrm{~h}$, age $\geq 60$ years old, and meeting the diagnostic criteria for acute respiratory failure(Tierney et al. 2020). Cases with missing data, advanced cancer, pregnancy, automatic discharge, and abandonment of treatment were excluded from the study. This study was approved by the Ethics Committee of the Second Affiliated Hospital of Wenzhou Medical University. IRB approval number: (2020) Ethical Approval No. 94.

\section{Statistics analysis}

Continuous data are presented as mean \pm standard deviation (SD) or median (IQR) according to the normal or non-normality distribution. Kolmogorov-Smirnov test was performed to determine normal distribution. Categorical variables were presented as frequency(proportion). Continuous data were compared using the Student t-test or Mann-Whitney U test and proportion variables were compared using chi-squared test or Fisher exact tests, as appropriate. For the development of the nomograms, the univariate and multivariable logistic regression were used to identify prognosis factors from the training data set. A nomogram was formulated based on the results of multivariable analysis, a final model selection was performed by a backward stepdown selection process with the Akaike information criterion(Harrell et al. 1996). The 'rms' package was used for nomogram and calibration curve(Liu et al. 2020). The accuracy of the nomogram to predict the 30-day mortality of ARF was quantified using the concordance index (C-index), which is equal to the area under the Receiver Operating Characteristic (ROC) curve and ranges from 0.5 to 1(Uno 
108 et al. 2011). The difference of C-index was compared by DeLong's non-parametric 109 approach(DeLong et al. 1988). The calibration of the model is assessed by the calibration curves 110 and determined using the Hosmer-Lemeshow goodness-of-fit test in the training set and validation 111 set(Grant et al. 2018). The 'rmda' package was performed for decision curve analysis (DCA) by 112 quantifying the net benefits to assess the clinical value of the model(Vickers \& Elkin 2006). We 113 did the statistics analyses and figures production using $\mathrm{R}$ software (version 3.6.1). All statistics 114 tests were two-sided, and $P$ values $<0.05$ were considered statistically significant.

\section{RESULTS}

\section{Patient characteristics}

117 A total of 1,432 patients were included in this study. Patients from MIMIC-III database (759 cases) were categorized into the training set, the 30 -day mortality in the training set were $38.6 \%$. The 673 patients from our hospital were categorized into the validation set, the 30 -day mortality was $40.5 \%$. The patient characteristics and laboratory findings of the training and validation sets are shown in Table 1 . There were no statistically significant differences between the training and validation set.

\section{Prognostic factors in the nomogram}

Baseline demographic, laboratory variables, including SOFA and APS-III score for the prediction of 30-day mortality were determined using univariate logistic regression firstly. The heart rate, respiratory rate, systolic pressure, spo2, bilirubin, albumin, lactate, APTT, PT, BUN and 24 h urine output were prognostic factors of 30-day mortality in univariate logistic regression analysis. All above statistically significant prognostic factors were entered into the multivariable logistic regression for adjusting the confounding factors for 30-day mortality. The heart rate, respiratory rate, systolic pressure, $\mathrm{SPO}_{2}$, albumin and $24 \mathrm{~h}$ urine output were independent prognostic factors for 30-day mortality (Table 2).

\section{Prognostic nomogram for 30-day mortality}

We established this nomogram, elderly ARF mortality prediction nomogram (e-ARF-MPN), which incorporated the significant prognostic factors from the multivariable analysis (Figure 1, S. Figure 1). This e-ARF-MPN had a C-index of 0.741 (95\% confidence interval [CI], 0.706-0.777) 
135 for predicting the 30-day mortality in ARF patients. Meanwhile, we developed two more 136 nomograms according to SOFA and APS-III scores (S. Figure 2). The SOFA nomogram had a C137 index of 0.653 (95\% CI: $0.613-0.693)$ and the APS-III nomogram had a C-index of 0.707 (95\% 138 CI: 0.670-0.744) (supplementary materials). Our e-ARF-MPN had a better predictive power than

139 140 141 142 143 144 145 146 147 148 149 150 both the SOFA $(P<0.001)$ and APS-III $(P=0.039)$ scores. For example, the calibration curve for eARF-MPN predicted 30-day mortality observed for in ARF patients in the training set was better than the SOFA and APS-III calibration curves (Figure 2). Hosmer-Lemeshow test showed nonsignificant statistic (chi-square $=9.132, P=0.2197$ ) in the training set.

\section{External validation of the nomogram}

The C-index of established e-ARF-MPN was 0.687 (95\%CI: 0.646-0.727) for predicting 30-day mortality in the validation set. The C-index of APS-III and SOFA were 0.677 (95\%CI: 0.635 $0.719)$ and 0.613 (95\%CI: $0.569-0.657)$, respectively. It has similar predictive power to the APSIII $(\mathrm{P}>0.05)$, but significantly higher than the SOFA $(P<0.001)$. The calibration curves revealed adequate fit of the e-ARF-MPN and APS-III predicting the 30-day mortality in the validation set, which is significantly better than the calibration curve for SOFA (Figure 3). Hosmer-Lemeshow test showed nonsignificant statistic (chi-square $=10.086, P=0.2025)$ in validation set.

\section{Decision Curve Analysis of the nomogram}

The decision curve analysis (DCA) showed that this e-ARF-MPN had a large threshold probability range than the SOFA and APS-III scores. For example, whether mechanical ventilation should be used in a patient with respiratory failure. The decision curve analysis of our nomogram showed that the nomogram assisted- mechanical ventilation decision adds more net benefit to respiratory failure patients than either the treat-all-patients (all patients treated with mechanical ventilation) or treat-none-patients (no patients treated with mechanical ventilation) when the threshold probability is more than $8 \%$. Compared to the net benefit of SOFA- and APS-III-assisted decisions, at the same threshold probability, e-ARF-MPN showed higher net benefit. It unveiled the clinical utility of proposed e-ARF-MPN. (Figure 4).

\section{DISCUSSION}


162 Nomogram is a visualization of regression analysis, which is widely used in clinical disease 163 diagnosis and prognosis evaluation (Callegaro et al. 2017; Chen et al. 2019; Wang et al. 2019; 164 Zhou et al. 2019). In this study, we developed and validated a novel e-ARF-MPN to predict the 165 mortality risk among elderly patients with ARF. Our results show that this e-ARF-MPN is mainly 166 based on vital signs and laboratory examination. The initial vital signs include heart rate, respiratory rate, systolic blood pressure, and $\mathrm{SpO}$, which were identified as independent predictors of mortality in elderly patients with ARF. With the increase of heart rate and respiratory rate, the risk of death increases. Furthermore, a decrease of systolic blood pressure and blood oxygen saturation will also increase the risk of death, both of which have a greater weight in the evaluation of short-term prognosis. Maintaining circulation stability and increasing blood oxygen saturation play an important role in reducing mortality of ARF in the elderly patients.

Currently, urinary output and serum creatinine are used to evaluate kidney function.

However, a study has shown that serum creatinine was an unreliable indicator of acute changes in renal function( $\mathrm{Li}$ et al. 2016). Our study also showed that urinary output was superior to serum creatinine in predicting short-term mortality of elderly patients with ARF. Although the assessment of AKI stage is not necessarily based on urine volume, the initial postoperative urine volume was considered an accurate predictor of delayed graft function(Kim et al. 2019). The reduction of urinary output can be attributed to insufficient blood flow to the kidneys, due to reduced blood volume and systolic pressure. Albumin, synthesized by the liver, is considered an important factor associated with malnutrition among patients. It tends to improve the microcirculatory performance which supports the maintenance of major organ functions(Liu et al. 2017). Thus, albumin was regarded as an important biomarker to evaluate the poor prognosis of hospitalized patients(Akirov et al. 2017). Our research also showed that the risk of death increased gradually with the decrease of plasma albumin. Therefore, plasma albumin may play an important role in predicting the mortality of elderly patients with ARF.

Finally, the e-ARF-MPN incorporates 6 items of heart rate, respiratory rate, systolic blood pressure, SpO2, urinary output, and plasma albumin. In order to prove the calibration of the 
nomogram, clinical data was collected from different institutions. As is well known, the internal validity associated with the explanation of the results, and the external validity related to the generalizability of the results (Hong et al. 2019; Huebschmann et al. 2019). Through the internal and external validation data set analysis, the calibration of our e-ARF-MPN has been proved to be highly consistent, which was more accurate than APS-III (B) and SOFA scores. At present, SOFA score has been widely used in assessment of critical diseases(Gole et al. 2020; Mebazaa et al. 2018), especially in the prognosis of multiple organ failure. When compared with APS-III and SOFA scores, nomogram developed in this study has fewer indicators but has better discrimination and calibration. This means that our nomogram may be popularized to predict the outcome of elderly patients with ARF.

However, evaluating the clinical usefulness of our nomogram depends on how much it benefits the patient, not just its popularization(Huang et al. 2016). DCA is a novel method that has been widely used in the evaluation of clinical research effectiveness(Hijazi et al. 2016; Prabhu et al. 2019; Talluri \& Shete 2016). It offers insight into clinical consequences on the basis of threshold probability, from which the net benefit could be derived(Balachandran et al. 2015). According to the DCA results, the application value of our nomogram is better than that of APS-III (B) and SOFA scores.

Our study has several limitations. First, our study was single center study and this e-ARF-MPN was only been validated in our hospital. We need to validate our e-ARF-MPN in broad external population. Second, our e-ARF-MPN is only applicable to the elderly ARF patients. Third, we reported 30-day all-cause mortality instead of ARF specific decease cause. Fourth, the time frame of our database collection i.e., from 2001 to 2012 is broad. Recent advance in the ARF treatment, i.e., noninvasive ventilation, high frequency oscillatory ventilation, and early intubation, might have a role in mortality.

\section{CONCLUSION:}

In conclusion, this study presents a novel nomogram that incorporates heart rate, respiratory rate, systolic blood pressure, SpO2, urinary output, and plasma albumin. It was better than both 
216

217

218

219

220

221

222

223

224

225

226

227

228

229

230

231

232

233

234

235

236

237

238

239

240

241

242

243

244

245

246

247

248

249

the APS-III (B) and SOFA scores; and could be useful as decision support on the prediction of mortality risk in elderly patients with ARF.

\section{List of abbreviations}

ARF: acute respiratory failure; MIMIC-III v1.4: Intensive Care III database version 1.4; SpO2: Percutaneous oxygen saturation; WBC: white blood cell; BUN: blood urea nitrogen; APTT: activated partial thromboplastin time; PT: prothrombine time; SOFA: sequential organ failure assessment score; APS-III: Acute Physiology Score III.

\section{REFERENCES}

Akirov A, Masri-Iraqi H, Atamna A, and Shimon I. 2017. Low Albumin Levels Are Associated with Mortality Risk in Hospitalized Patients. Am J Med 130:1465 e1411-1465 e1419. 10.1016/j.amjmed.2017.07.020

Balachandran VP, Gonen M, Smith JJ, and DeMatteo RP. 2015. Nomograms in oncology: more than meets the eye. Lancet Oncol 16:e173-180. 10.1016/S1470-2045(14)71116-7

Behrendt CE. 2000. Acute respiratory failure in the United States: incidence and 31-day survival. Chest 118:11001105. 10.1378/chest.118.4.1100

Callegaro D, Miceli R, Mariani L, Raut CP, and Gronchi A. 2017. Soft tissue sarcoma nomograms and their incorporation into practice. Cancer 123:2802-2820. 10.1002/cncr.30721

Chen Z, Lin RM, Bai YK, and Zhang Y. 2019. Establishment and Verification of Prognostic Nomograms for Patients with Gastrointestinal Stromal Tumors: A SEER-Based Study. Biomed Res Int 2019:8293261. $10.1155 / 2019 / 8293261$

DeLong ER, DeLong DM, and Clarke-Pearson DL. 1988. Comparing the areas under two or more correlated receiver operating characteristic curves: a nonparametric approach. Biometrics 44:837-845.

Dong F, Shen Y, Gao F, Shi X, Xu T, Wang X, Zhang X, Zhong S, Zhang M, Chen S, and Shen Z. 2018. Nomograms to Predict Individual Prognosis of Patients with Primary Small Cell Carcinoma of the Bladder. $J$ Cancer 9:1152-1164. 10.7150/jca.23344

Flaatten H, de Lange DW, Artigas A, Bin D, Moreno R, Christensen S, Joynt GM, Bagshaw SM, Sprung CL, Benoit D, Soares M, and Guidet B. 2017. The status of intensive care medicine research and a future agenda for very old patients in the ICU. Intensive Care Medicine 43:1319-1328. 10.1007/s00134-017-4718-z

Gilbride L, Siker M, Bovi J, Gore E, Schultz C, and Hall WA. 2018. Current Predictive Indices and Nomograms To 
250

251

252

253

254

255

256

257

258

259

260

261

262

263

264

265

266

267

268

269

270

271

272

273

274

275

276

277

278

279

280

281

282

283

284

285

286

287

288

289

290

Enable Personalization of Radiation Therapy for Patients With Secondary Malignant Neoplasms of the Central Nervous System: A Review. Neurosurgery 82:595-603. 10.1093/neuros/nyx631

Gole AR, Srivastava SL, and Neeraj. 2020. Prognostic accuracy of SOFA score, SIRS criteria, NEWS and MEWS scores for in-hospital mortality among adults admitted to ICU with suspected sepsis. J Assoc Physicians India 68:87.

Grant SW, Collins GS, and Nashef SAM. 2018. Statistical Primer: developing and validating a risk prediction model. Eur J Cardiothorac Surg 54:203-208. 10.1093/ejcts/ezy180

Harrell FE, Jr., Lee KL, and Mark DB. 1996. Multivariable prognostic models: issues in developing models, evaluating assumptions and adequacy, and measuring and reducing errors. Stat Med 15:361-387. 10.1002/(sici)10970258(19960229)15:4<361::Aid-sim168>3.0.Co;2-4

Hijazi Z, Oldgren J, Lindback J, Alexander JH, Connolly SJ, Eikelboom JW, Ezekowitz MD, Held C, Hylek EM, Lopes RD, Siegbahn A, Yusuf S, Granger CB, Wallentin L, Aristotle, and Investigators R-L. 2016. The novel biomarker-based $\mathrm{ABC}$ (age, biomarkers, clinical history)-bleeding risk score for patients with atrial fibrillation: a derivation and validation study. Lancet 387:2302-2311. 10.1016/S0140-6736(16)00741-8

Hong JY, You JS, Kim MJ, Lee HS, Park YS, Chung SP, and Park I. 2019. Development and external validation of new nomograms by adding ECG changes (ST depression or tall $\mathrm{T}$ wave) and age to conventional scoring systems to improve the predictive capacity in patients with subarachnoid haemorrhage: a retrospective, observational study in Korea. BMJ Open 9:e024007. 10.1136/bmjopen-2018-024007

Huang YQ, Liang CH, He L, Tian J, Liang CS, Chen X, Ma ZL, and Liu ZY. 2016. Development and Validation of a Radiomics Nomogram for Preoperative Prediction of Lymph Node Metastasis in Colorectal Cancer. J Clin Oncol 34:2157-2164. 10.1200/JCO.2015.65.9128

Huebschmann AG, Leavitt IM, and Glasgow RE. 2019. Making Health Research Matter: A Call to Increase Attention to External Validity. Annu Rev Public Health 40:45-63. 10.1146/annurev-publhealth-040218-043945

Johnson AE, Pollard TJ, Shen L, Lehman LW, Feng M, Ghassemi M, Moody B, Szolovits P, Celi LA, and Mark RG. 2016. MIMIC-III, a freely accessible critical care database. Sci Data 3:160035. 10.1038/sdata.2016.35

Kim J, Pyeon T, Choi JI, Kang JH, Song SW, Bae HB, and Jeong S. 2019. A retrospective study of the relationship between postoperative urine output and one year transplanted kidney function. BMC Anesthesiol 19:231. 10.1186/s12871-019-0904-6

Li WH, Wang L, He HY, Chen J, and Yu YR. 2016. Expression of neutrophil gelatinase-associated lipocalin in low osmolar contrast-induced nephropathy in rats and the effect of N-acetylcysteine. Exp Ther Med 12:31753180. 10.3892/etm.2016.3779

Liu J, Nie S, Li S, Meng H, Sun R, Yang J, and Cheng W. 2020. Methylation-driven genes and their prognostic value in cervical squamous cell carcinoma. Ann Transl Med 8:868. 10.21037/atm-19-4577

Liu Y, Yang W, and Wei J. 2017. Guiding Effect of Serum Procalcitonin (PCT) on the Antibiotic Application to Patients with Sepsis. Iran J Public Health 46:1535-1539.

Lloyd-Sherlock P, McKee M, Ebrahim S, Gorman M, Greengross S, Prince M, Pruchno R, Gutman G, Kirkwood T, O'Neill D, Ferrucci L, Kritchevsky SB, and Vellas B. 2012. Population ageing and health. Lancet 379:12951296. 10.1016/s0140-6736(12)60519-4

Mebazaa A, Geven C, Hollinger A, Wittebole X, Chousterman BG, Blet A, Gayat E, Hartmann O, Scigalla P, Struck J, Bergmann A, Antonelli M, Beishuizen A, Constantin JM, Damoisel C, Deye N, Di Somma S, Dugernier T, Francois B, Gaudry S, Huberlant V, Lascarrou JB, Marx G, Mercier E, Oueslati H, Pickkers P, Sonneville 
R, Legrand M, Laterre PF, and Adren OSSsi. 2018. Circulating adrenomedullin estimates survival and reversibility of organ failure in sepsis: the prospective observational multinational Adrenomedullin and Outcome in Sepsis and Septic Shock-1 (AdrenOSS-1) study. Crit Care 22:354. 10.1186/s13054-018-2243-2

Prabhu V, Rosenkrantz AB, Otazo R, Sodickson DK, and Kang SK. 2019. Population net benefit of prostate MRI with high spatiotemporal resolution contrast-enhanced imaging: A decision curve analysis. J Magn Reson Imaging 49:1400-1408. 10.1002/jmri.26318

Ray P, Birolleau S, Lefort Y, Becquemin MH, Beigelman C, Isnard R, Teixeira A, Arthaud M, Riou B, and Boddaert J. 2006. Acute respiratory failure in the elderly: etiology, emergency diagnosis and prognosis. Crit Care 10:R82. 10.1186/cc4926

Talluri R, and Shete S. 2016. Using the weighted area under the net benefit curve for decision curve analysis. BMC Med Inform Decis Mak 16:94. 10.1186/s12911-016-0336-x

Tierney DM, Huelster JS, Overgaard JD, Plunkett MB, Boland LL, St Hill CA, Agboto VK, Smith CS, Mikel BF, Weise BE, Madigan KE, Doshi AP, and Melamed RR. 2020. Comparative Performance of Pulmonary Ultrasound, Chest Radiograph, and CT Among Patients With Acute Respiratory Failure. Crit Care Med 48:151-157. 10.1097/ccm.0000000000004124

Uno H, Cai T, Pencina MJ, D'Agostino RB, and Wei LJ. 2011. On the C-statistics for evaluating overall adequacy of risk prediction procedures with censored survival data. Stat Med 30:1105-1117. 10.1002/sim.4154

Vickers AJ, and Elkin EB. 2006. Decision curve analysis: a novel method for evaluating prediction models. Med Decis Making 26:565-574. 10.1177/0272989x06295361

Wang Y, Sun K, Shen J, Li B, Kuang M, Cao Q, and Peng S. 2019. Novel Prognostic Nomograms Based on Inflammation-Related Markers for Patients with Hepatocellular Carcinoma Underwent Hepatectomy. Cancer Res Treat 51:1464-1478. 10.4143/crt.2018.657

Zhou Z, Mo S, Dai W, Xiang W, Han L, Li Q, Wang R, Liu L, Zhang L, Cai S, and Cai G. 2019. Prognostic nomograms for predicting cause-specific survival and overall survival of stage I-III colon cancer patients: a large population-based study. Cancer Cell Int 19:355. 10.1186/s12935-019-1079-4

Figure 1. Nomogram to predicted 30-day mortality in ARF patients. The nomogram was developed in the training set, with the heart rate, respiratory rate, systolic pressure, SPO2, albumin, and $24 \mathrm{~h}$ urine output incorporated.

Figure 2. Calibration curves of the nomogram (A), APS-III (B), and SOFA (C) predicted 30-day mortality in training set. Calibration curve represents the calibration of the nomogram, which shows the consistency between the predicted 30-day mortality and actual 30-day mortality of ARF patients. Y-axis represents the actual 30-day 
327

328

329

330

331

332

333

334

335

336

337

338

339

340

341

342 mortality, X-axis represents the predicted 30-day mortality. The diagonal line represents a perfect prediction by an ideal model, and black solid line represents the prediction performance of the nomogram, of which a closer fit to the diagonal line means a better prediction.

Figure 3. Calibration curves of the nomogram (A), APS-III (B), and SOFA (C) predicted 30-day mortality in validation set. Y-axis represents the actual 30-day mortality, X-axis represents the predicted 30-day mortality. Black solid line represents the prediction performance of the nomogram, the diagonal line represents an ideal nomogram model. The diagonal line represents a perfect prediction by an ideal model, and black solid line represents the prediction performance of the nomogram, of which a closer fit to the diagonal line means a better prediction.

Figure 4. Decision curve for the training set cohort implicating the net benefit with respect to the use of the nomogram, APS-III, and SOFA score for predicting 30-day in ARF patients. The Y-axis represents the net benefit. The X-axis represents the threshold value. The light grey line represents the assumption that all patients have the outcome (dead). Thin black line represents the assumption that no patients have the outcome (dead). 


\section{Figure 1}

Figure 1.

Figure 1. Nomogram to predicted 30-day mortality in ARF patients. The nomogram was developed in the training set, with the heart rate, respiratory rate, systolicpressure, SPO2, albumin, and $24 \mathrm{~h}$ urine output incorporated. 
Points

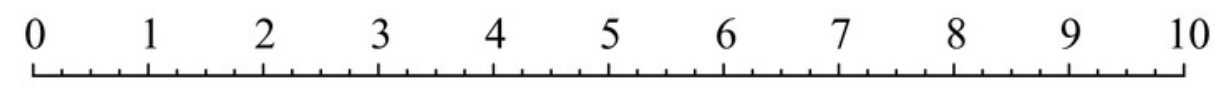

Heart rate

$$
\underset{\leq 100 \quad>100 \sim \leq 120}{\stackrel{>}{>}=120}
$$

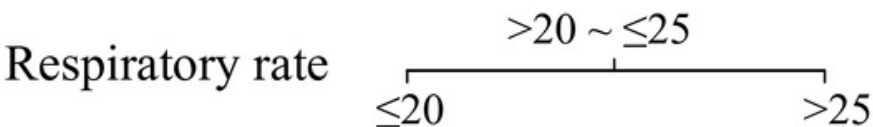

Systolic pressure $\begin{array}{ll}\geq 70 \sim<90 & <60 \\ & \geq 90 \quad>60 \sim \leq 70\end{array}$

SPO2

$\begin{array}{lllllllllll}100 & 95 & 90 & 85 & 80 & 75 & 70 & 65 & 60 & 55 & 50\end{array}$

Albumin

$$
\begin{array}{llllllllllllll}
\hline 3.6 & 3.4 & 3.2 & 3 & 2.8 & 2.6 & 2.4 & 2.2 & 2 & 1.8 & 1.6 & 1.4 & 1.2 & 1
\end{array}
$$

Urine output

$$
\geq 1000<400 \sim<1000
$$

Total Points

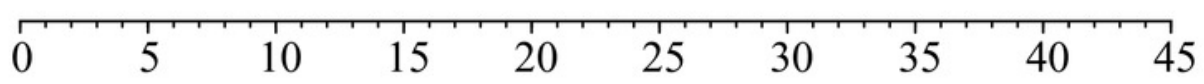

30-day mortality

$$
\begin{array}{llllllllll}
\hline 0.1 & 0.2 & 0.3 & 0.4 & 0.5 & 0.6 & 0.7 & 0.8 & 0.9 & 0.95
\end{array}
$$


Figure 2

Figure 2

Figure 2. Calibration curves of the nomogram (A), APS-III (B) and SOFA (C) predicted 30-day mortality in training set. Calibration curve represents the calibration of the nomogram, which shows the consistency between the predicted 30-day mortality and actual 30-day mortality of ARF patients. Y-axis represents the actual 30-day mortality, X-axis represents the predicted 30-day mortality. The diagonal line represents a perfect prediction by an ideal model, and black solid line represents the prediction performance of the nomogram, of which a closer fit to the diagonal line means a better prediction.
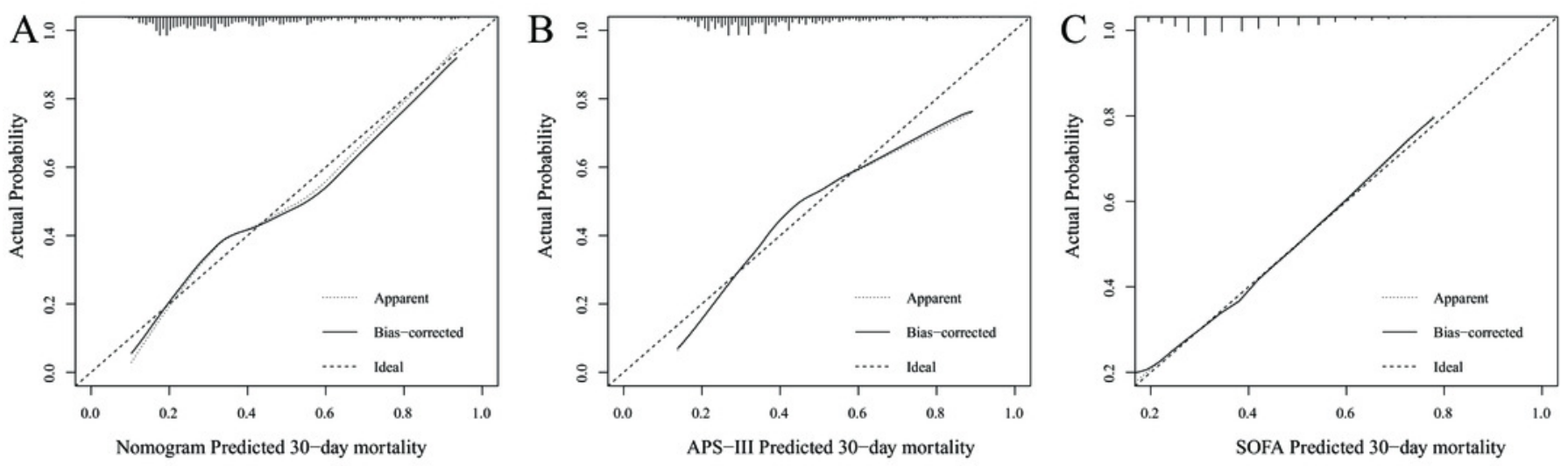


\section{Figure 3}

Figure 3

Figure 3. Calibration curves of the nomogram (A), APS-III (B) and SOFA (C) predicted 30-day mortality in validation set. Y-axis represents the actual 30-day mortality, X-axis represents the predicted 30-day mortality. Black solid line represents the prediction performance of the nomogram, the diagonal line represents an ideal nomogram model. The diagonal line represents a perfect prediction by an ideal model, and black solid line represents the prediction performance of the nomogram, of which a closer fit to the diagonal line means a better prediction.
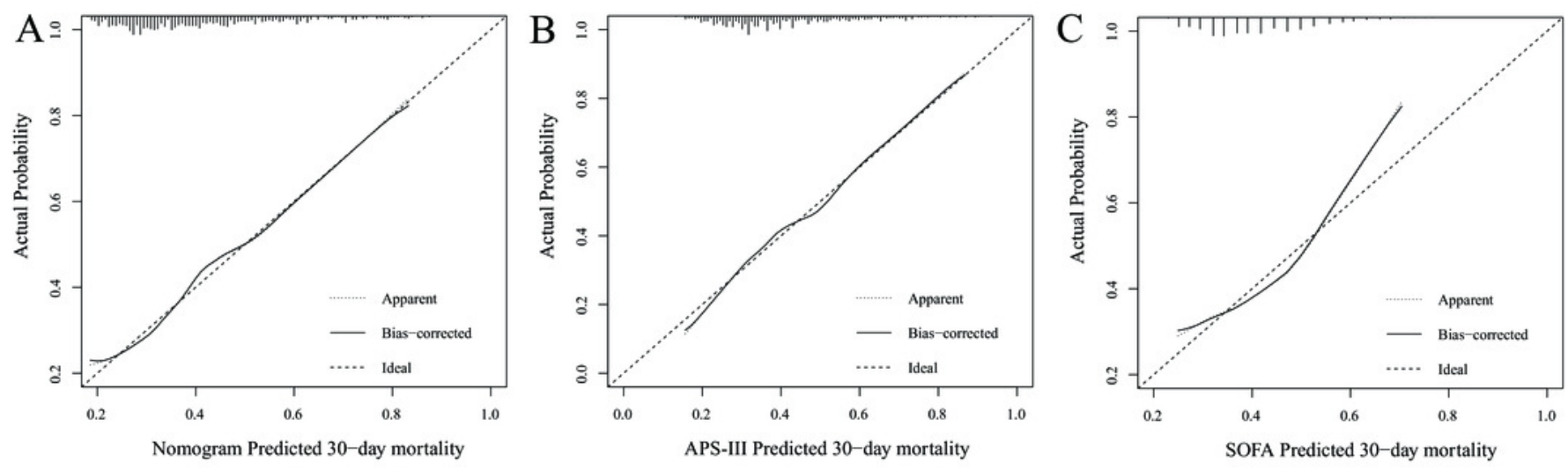


\section{Figure 4}

Figure 4

Figure 4. Decision curve for the training set cohort implicating the net benefit with respect to the use of the nomogram, APS-III, and SOFA score for predicting 30-day in ARF patients. The Y-axis represents the net benefit. The $\mathrm{X}$-axis represents the threshold value. The red line represents the nomogram model, blue line represents the APS-III score and green line represents the SOFA score. The light grey line represents the assumption that all patients have the outcome (dead). Thin black line represents the assumption that no patients have the outcome (dead). 


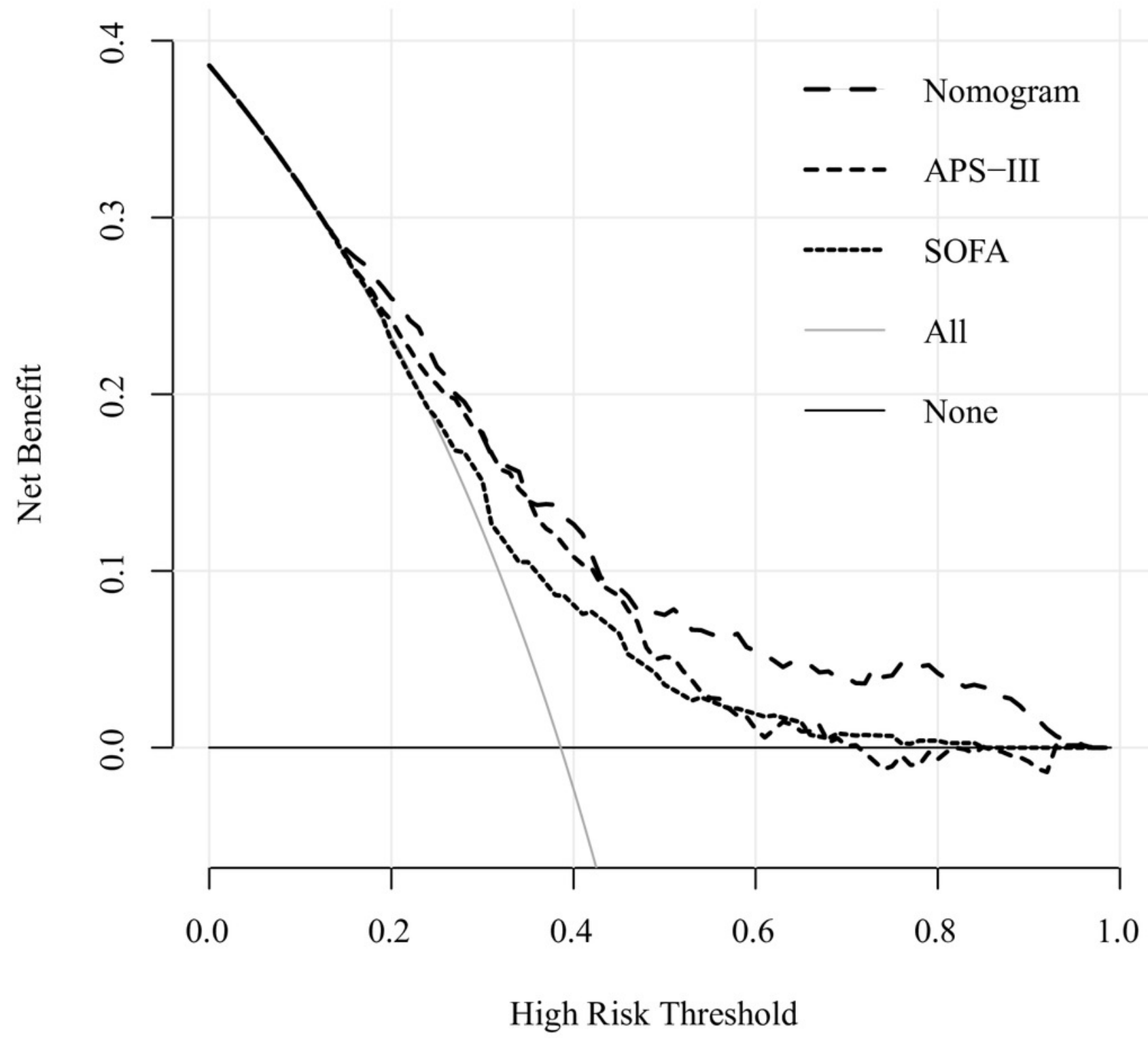




\section{Table 1 (on next page)}

Patient characteristics in training and validation set. 
1

2 Table 1 Patient characteristics in training and validation set.

\begin{tabular}{|c|c|c|c|}
\hline Variables & $\begin{array}{l}\text { Training set } \\
(\mathrm{n}=759)\end{array}$ & $\begin{array}{l}\text { Validation set } \\
\qquad(\mathrm{n}=673)\end{array}$ & P-value \\
\hline Age, median (IQR) & $76(68,83)$ & $74(66,82)$ & 0.005 \\
\hline Gender, male, n (\%) & $395(52.0)$ & $384(57.1)$ & 0.064 \\
\hline \multicolumn{4}{|l|}{ Vital signs } \\
\hline Temperature, Median $(\mathrm{IQR})\left({ }^{\circ} \mathrm{C}\right)$ & $37.6(37.08,38.25)$ & $37.44(36.89,38.06)$ & $<0.001$ \\
\hline Heart rate, Median (IQR) (bmp) & $109(95,124.5)$ & $107(92,122)$ & 0.021 \\
\hline Systolic pressure, Median (IQR) (mmHg) & $82(72,92)$ & $84(73,95)$ & 0.013 \\
\hline Respiratory rate, Median (IQR) (per minute) & $28(24,33)$ & $28(24,33)$ & 0.748 \\
\hline $\mathrm{SPO}_{2}$, Median (IQR) (\%) & $92(88,95)$ & $92(88,95)$ & 0.965 \\
\hline \multicolumn{4}{|l|}{ Laboratory findings } \\
\hline WBC, Median $(\mathrm{IQR})\left(10^{\wedge 9} / \mathrm{L}\right)$ & $15.5(10.6,21.4)$ & $14(10,19.7)$ & 0.002 \\
\hline Hemoglobin, Median $(\mathrm{IQR})\left(10^{\wedge 9} / \mathrm{L}\right)$ & $9.6(8.4,11)$ & $9.8(8.5,11.1)$ & 0.279 \\
\hline Platelet, Median (IQR) $\left(10^{\wedge 9} / \mathrm{L}\right)$ & $181(114.5,250.5)$ & $178(115,250)$ & 0.846 \\
\hline Albumin, Median (IQR) (g/dL) & $2.9(2.4,3.4)$ & $3(2.5,3.4)$ & $<0.001$ \\
\hline Bilirubin, Median (IQR) (mg/dL) & $0.7(0.4,1.35)$ & $0.7(0.4,1.4)$ & 0.382 \\
\hline Creatinine, Median (IQR) (mg/dL) & $1.5(1,2.4)$ & $1.5(1,2.6)$ & 0.323 \\
\hline Glucose, Median (IQR) (mg/dL) & $107(88,133)$ & $109(90,133)$ & 0.293 \\
\hline BUN, Median (IQR) (mg/dL) & $35(23,55)$ & $35(22,56)$ & 0.956 \\
\hline APTT, Median (IQR) (s) & $36.3(29,54.95)$ & $35.7(28.6,54.6)$ & 0.203 \\
\hline PT, Median (IQR) (s) & $15.4(13.8,18.5)$ & $15.6(13.8,20.3)$ & 0.062 \\
\hline Lactate, Median (IQR) $(\mathrm{mmol} / \mathrm{L})$ & $2.7(1.6,5.15)$ & $2.3(1.5,4.1)$ & $<0.001$ \\
\hline \multicolumn{4}{|l|}{ Severity score } \\
\hline SOFA, Median (IQR) & $6(4,9)$ & $7(4,10)$ & 0.053 \\
\hline APS-III, Median (IQR) & $58(45,76)$ & $60(46,81)$ & 0.159 \\
\hline
\end{tabular}


24 h Urine output, Median (IQR) (ml)

Mechanical ventilation, $n(\%)$

Length of stay, Median (IQR)

30-day mortality, n (\%)

$$
\begin{gathered}
1242(676.5,2112.5) \\
619(81.6) \\
5.07(2.59,11.59) \\
293(38.6)
\end{gathered}
$$

$$
\begin{gathered}
1195(625,1950) \\
545(81.0) \\
5.18(2.55,10.09) \\
273(40.6)
\end{gathered}
$$

0.834

0.818

0.482

3 SpO2, percutaneous oxygen saturation; WBC, white blood cell; BUN, blood urea nitrogen; APTT, activated

4 partial thromboplastin time; PT, prothrombine time; SOFA, sequential organ failure assessment, APS-III, Acute 5 Physiology Score III.

6 


\section{Table 2 (on next page)}

The prognostic factors of 30-day mortality in univariate and multivariable logistic analyses 
1 Table 2 The prognostic factors of 30-day mortality in univariate and multivariable logistic analyses

\begin{tabular}{lcccccc}
\hline Variables & \multicolumn{3}{c}{ univariate } & \multicolumn{3}{c}{ multivariable } \\
\cline { 2 - 6 } & OR & $95 \% \mathrm{CI}$ & $P$-value & OR & $95 \% \mathrm{CI}$ & $P$-value \\
\hline Heart rate & & & & & & \\
$\leq 100$ & reference & - & - & reference & - & - \\
$\square 100 \sim \leq 120$ & 1.53 & $1.07-2.21$ & $\mathbf{0 . 0 2 1 8}$ & 1.25 & $0.84-1.89$ & 0.269 \\
$\square 120$ & 2.12 & $1.47-3.05$ & $<\mathbf{0 . 0 0 1}$ & 1.53 & $1.01-2.33$ & $\mathbf{0 . 0 4 6}$
\end{tabular}

Systolic pressure

$\begin{array}{lrccccc}\geq 90 & \text { reference } & - & - & \text { reference } & - & - \\ \geq 70 \sim \square 90 & 1.65 & 1.15-2.37 & \mathbf{0 . 0 0 7} & 1.10 & 0.74-1.63 & 0.629 \\ \geq 60 \sim \square 70 & 3.54 & 2.194-5.88 & <\mathbf{0 . 0 0 1} & 1.77 & 1.01-3.13 & \mathbf{0 . 0 4 8} \\ \square 60 & 7.84 & 4.42-14.38 & <\mathbf{0 . 0 0 1} & 3.68 & 1.91-7.28 & <\mathbf{0 . 0 0 1}\end{array}$

Respiratory rate

\begin{tabular}{|c|c|c|c|c|c|c|}
\hline$\leq 20$ & reference & - & - & reference & - & - \\
\hline$\square 20 \sim \leq 25$ & 1.18 & $0.61-2.41$ & 0.619 & 1.31 & $0.64-2.79$ & 0.475 \\
\hline$\square 25$ & 2.29 & $1.25-4.46$ & 0.01 & 2.1 & $1.13-3.34$ & 0.036 \\
\hline $\mathrm{SPO}_{2}$ & 0.96 & $0.94-0.97$ & $<0.001$ & 0.97 & 0.96-0.99 & 0.005 \\
\hline Albumin & 0.45 & $0.35-0.58$ & $<0.001$ & 0.61 & $0.45-0.81$ & $<0.001$ \\
\hline \multicolumn{7}{|l|}{ Urine output } \\
\hline$\geq 1000$ & reference & - & - & reference & - & - \\
\hline$\geq 400 \sim \square 1000$ & 2.52 & $1.78-3.58$ & $<0.001$ & 2.11 & $1.44-3.09$ & $<0.001$ \\
\hline$\square 400$ & $5.20-$ & $3.32-8.27$ & $<0.001$ & 2.56 & $1.51-4.38$ & $<0.001$ \\
\hline
\end{tabular}

$2 \mathrm{SpO}$, percutaneous oxygen saturation 